\title{
Self-Seeding of a Gain-Switched Integrated Dual-Laser Source for the Generation of Highly Wavelength-Tunable Picosecond Optical Pulses
}

\author{
P. Anandarajah, P. J. Maguire, A. Clarke, and L. P. Barry
}

\begin{abstract}
The authors demonstrate the generation of nearly transform-limited optical pulses that are wavelength tunable over almost $50 \mathrm{~nm}$. The wide tuning range is obtained by self-seeding a gain-switched source containing two Fabry-Perot lasers, and employing a widely tunable Bragg grating in the feedback loop. The generated pulses exhibit Side-mode suppression ratios of $50 \mathrm{~dB}$ above and across the full tuning range.
\end{abstract}

Index Terms-Optical fiber communications, optical pulse generation, self-seeding, semiconductor laser, wavelength tunable.

\section{INTRODUCTION}

$\mathbf{T}$ 1 HE USE OF wavelength tunability as a means of providing dynamic provisioning, in next-generation photonic systems, is currently a key area that is attracting much attention [1]. This interest could be attributed to the convergence of data networking with multiwavelength optical networking, a natural outcome driven by the impending needs of the Internet. In addition to this development, current trends and technology maturity favor the deployment of optical communication systems, operating at line rates $40 \mathrm{~Gb} / \mathrm{s}$ and beyond, thereby making it more likely that return-to-zero (RZ) coding be used for data transmission, as it is easier to compensate for dispersion and nonlinear effects in the fiber by employing soliton-like propagation [2]. Taking into account these moves toward tunable optical systems employing RZ coding, it is obvious that the development of a wavelength tunable source of short optical pulses will be of paramount importance for future wavelength division multiplexed (WDM), optical time division multiplexed (OTDM), and hybrid WDM/OTDM optical communication systems [3].

One of the simplest and most reliable techniques available to generate wavelength-tunable picosecond optical pulses involves the self-seeding of a gain-switched Fabry-Perot (FP) laser, and many experimental schemes have been reported [4], [5]. Selfseeding entails the use of a wavelength selective external cavity to reinject a small fraction of the output light back into the gain-switched FP laser at only one longitudinal mode frequency. Provided that the optical signal reinjected into the laser arrives during the buildup of an optical pulse in the FP laser, then a single-moded output pulse is obtained. An important charac-

Manuscript received August 15, 2003; revised October 6, 2003. This work was supported in part by the Science Foundation Ireland and by Enterprise Ireland.

The authors are with the Research Institute for Networks and Communications Engineering, School of Electronic Engineering, Dublin City University, Dublin 9, Ireland (e-mail: barryl@eeng.dcu.ie).

Digital Object Identifier 10.1109/LPT.2003.821252

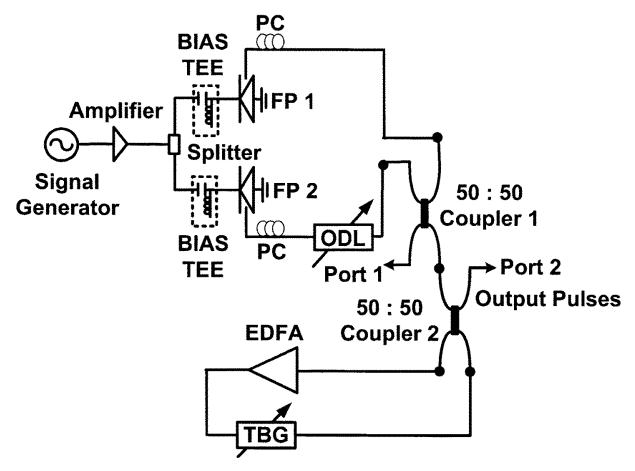

Fig. 1. Experimental setup for the generation of widely tunable optical pulses using the SSGS FP lasers.

teristic of these Self-Seeded Gain-Switched (SSGS) sources is the variation in the side-mode suppression ratio (SMSR) as the wavelength is tuned [6], as this may ultimately affect their usefulness in optical communication systems. In particular, recent work has demonstrated that, as the number of channels in a WDM system using SSGS pulse sources increases, the specifications on the required SMSR, due to cross-channel interference, may become very stringent [7]. It is thus likely that SMSRs in excess of $30 \mathrm{~dB}$ will be required for error-free operation of such systems.

Recent work in the development of wavelength tunable SSGS pulse sources has resulted in the generation of 90-130-ps optical pulses with SMSRs of around $32 \mathrm{~dB}$ that are tunable over 19-26 nm [8], [9]. The tunability of these systems was limited by factors such as the tunable range of the fiber Bragg grating (FBG) and the gain profile of the laser. In this letter, we show the generation of shorter pulses ( $\sim 20 \mathrm{ps})$ that exhibit SMSRs greater than $50 \mathrm{~dB}$ over a tuning range approaching $50 \mathrm{~nm}$. Our technique is based on the self-seeding of a gain-switched source containing two FP lasers, and the use of a widely tunable Bragg grating (TBG) filter. As we use two FP lasers with different gain curves, we can achieve a very large wavelength tuning range, and the high SMSR is essentially obtained by passing the self-seeded gain-switched pulses through the Bragg filter before the output.

\section{EXPERIMENTAL SETUP}

Fig. 1 illustrates the experimental configuration. It essentially consists of two gain switched FP laser diodes that are self-seeded using a single external cavity. The two FP lasers were chosen in such a way as to ensure that there was only 
a very small overlap between their gain profiles, and finer placement of the gain profiles of the two lasers could be achieved by temperature controlling the diodes. The peak of the gain curve for FP 1 is at $1524 \mathrm{~nm}$, while the peak of the gain curve for FP 2 is at $1561 \mathrm{~nm}$. The incorporation of two gain-switched FP lasers enhances the wavelength tuning range that can be achieved using this self-seeding setup, as will be demonstrated below.

The FP lasers used were commercial $1.5-\mu \mathrm{m}$ InGaAsP devices with threshold currents of approximately $26 \mathrm{~mA}$, and mode spacings of $1.12 \mathrm{~nm}$. Gain switching of both lasers was carried out by applying a dc-bias current of $17 \mathrm{~mA}$ and a 2.5-GHz sinusoidal modulation signal with a power of $29 \mathrm{dBm}$ to both devices. The gain-switched output from both lasers were then coupled together before the composite signal was fed into an external loop cavity, which was used to self-seed both lasers. The external cavity contained a polarization controller (PC), a 3-dB coupler, a TBG (bandwidth: $0.23 \mathrm{~nm}$, wavelength tuning range: $1460-1575 \mathrm{~nm}$, rejection ratio: $40 \mathrm{~dB}$, insertion loss: $5 \mathrm{~dB}$ ) and an erbium-doped fiber amplifier (EDFA). An optical isolator in the EDFA ensures that light only propagates in one direction around the feedback loop, and the tunable filter eliminates unwanted amplified spontaneous emission from the EDFA in addition to selecting the laser mode to be seeded. The external cavity for self-seeding FP 2 also contains a tunable optical delay line (just at the output of the laser) to ensure that simultaneous self-seeding of FP 1 and FP 2 can be achieved. The EDFA in the external cavity is required to overcome the high losses obtained in the TBG (which has an insertion loss of approximately $5 \mathrm{~dB}$ across the tuning range and a very narrow linewidth compared to the spectral width $(\sim 63 \mathrm{GHz})$ of the modes from the gain-switched laser), and ensure that there is sufficient light reinjected into either laser to obtain suitable SMSRs on the output pulses.

To achieve optimum SSGS pulse generation, the Bragg grating was initially tuned to one of the longitudinal modes of the gain-switched FP-1 laser. The frequency of the modulation was then varied to ensure that the signal reinjected into the laser, from the external cavity, arrives as an optical pulse is building up in the laser. An operating frequency of $2.498 \mathrm{GHz}$ was found to be suitable. The grating was then tuned to one of the longitudinal modes of FP 2 and, in this case, the tunable optical delay line was varied to ensure that the signal fed back into FP 2 arrives at the correct instant. The bias currents of FP 1 and FP 2 were then slightly changed to obtain the minimum pulsewidth. By subsequently tuning the grating across the gain curves of the lasers, we can achieve single-moded optical pulses over a very wide range of wavelengths. The output pulses, from the return arm of the second 50:50 fiber coupler (port 2) were characterized using a $50-\mathrm{GHz}$ photodiode in conjunction with a $50-\mathrm{GHz}$ digitizing oscilloscope, and a $0.05-\mathrm{nm}$ resolution spectrum analyzer.

\section{RESULTS AND DISCUSSION}

The optical spectrum of the dual-wavelength signal from the gain-switched lasers without self-seeding is shown in Fig. 2(a). It can clearly be seen that, by combining the output of the gain
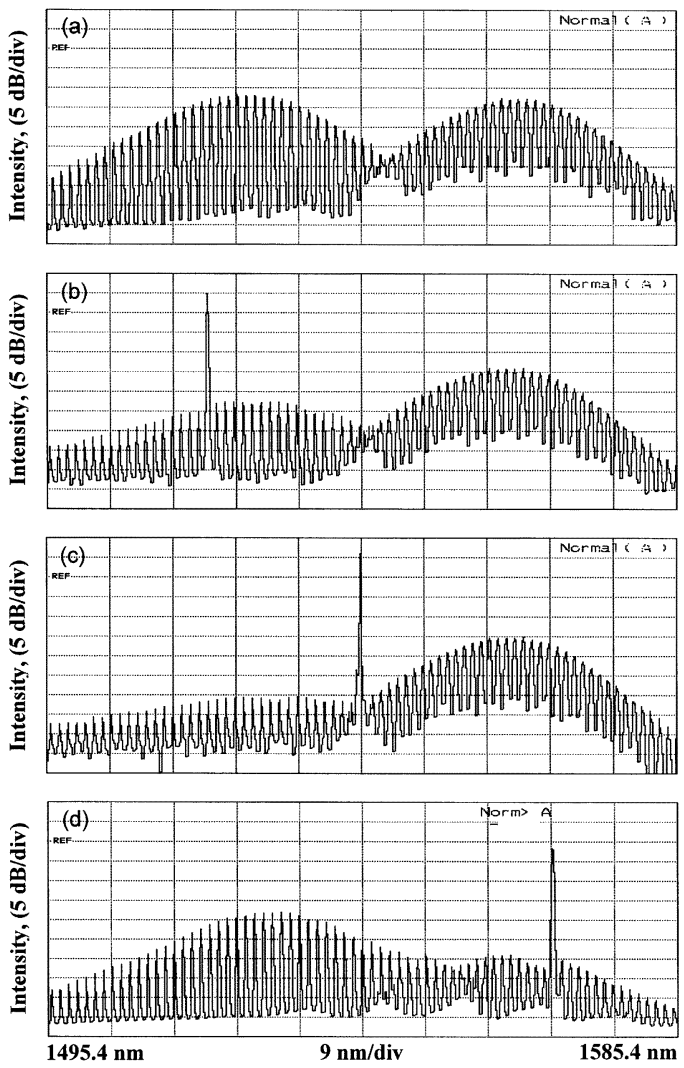

Fig. 2. Output optical spectra at port 1 of: (a) dual-wavelength signal. (b) Shortest wavelength that can be obtained: $1517.73 \mathrm{~nm}$. (c) Central wavelength at $1540.4 \mathrm{~nm}$. (d) Longest wavelength that can be obtained: $1566.64 \mathrm{~nm}$.

switched lasers in the wavelength domain, the composite span of the gain profile that could be used for seeding has been greatly increased. The gain spectra of the two lasers overlap at approximately $16 \mathrm{~dB}$ down from the peak of their gain curves. This overlap corresponds to the maximum wavelength of FP 1 , and the minimum wavelength of FP 2, for which we can achieve suitable SMSRs using the self-seeding configuration.

Different longitudinal modes of each FP laser were selectively excited when the seeding wavelength was tuned near the center of any desired mode. To obtain more continuous wavelength tuning with this setup, it is possible to use temperature tuning of the diodes in conjunction with tuning the Bragg grating. Fig. 2(b)-(d) shows in respective order the shortest, central, and longest wavelengths that could be seeded. The seeded spectra shown are the composite output (port 1) of the two SSGS lasers before passing through the optical filter and amplifier to be output to port 2 .

By taking the output pulses at port 2, we thus pass the composite signal from the SSGS lasers through the external cavity before being outputted. The effect of this is to eliminate the signal from the unseeded laser, and greatly improve the SMSR of the generated optical pulses from the seeded laser [as shown below in Fig. 3(b) and (d)]. The output pulses, and their associated spectra, generated at two specific wavelengths (1524 and $1560 \mathrm{~nm}$ ) are shown in Fig. 3. The deconvolved pulsewidth for the 1524-nm signal was $16 \mathrm{ps}$, while that of the $1560-\mathrm{nm}$ signal was $18.5 \mathrm{ps}$. The associated spectral widths of these two 

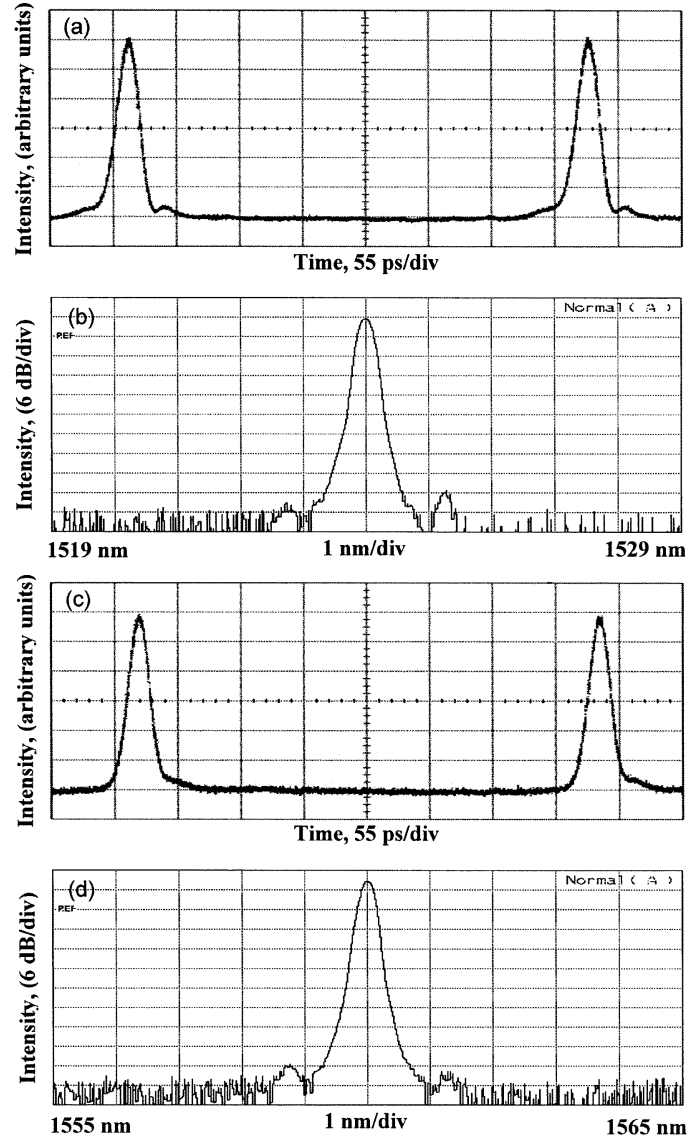

Fig. 3. (a) and (c) Output optical pulses and (b) and (d) associated spectra from port 2 for signal generation at $1524 \mathrm{~nm}$ [(a) and (b)] and $1560 \mathrm{~nm}$ [(c) and (d)]

signals were 27 and $26 \mathrm{GHz}$, respectively, while the SMSR of the generated pulses were 54 and $56 \mathrm{~dB}$, respectively. The measured pulsewidth remained reasonably constant (16-20 ps) as the output pulses were tuned across the entire tuning range, with slight increases at the limits of tunability, and the time-bandwidth product of the generated pulses remains in the 0.43-0.49 range over the tuning range [which is close to that of a transform limited Gaussian pulses (0.44)]. The average output power of the optical pulses is approximately $1.8 \mathrm{~mW}$. The main limitation on the wavelength tuning of the generated pulses was imposed by the gain bandwidth of the EDFA used in our experimental setup.

The dependence of the SMSR on the seeding wavelength was plotted and is shown in Fig. 4. It can be clearly seen that we were able to obtain an SMSR of $50 \mathrm{~dB}$ and above within a range of $48.91 \mathrm{~nm}(1517.73-1566.64 \mathrm{~nm})$. As the seeding power was increased, due to higher pump powers from the EDFA, the achievable SMSR was enhanced and the possible tuning range became wider; however, pulse deformation and instabilities were observed. The results shown are taken at the optimum level of EDFA pump power (around $20 \mathrm{~mW}$ ) to ensure maximum SMSR is achieved without pulse deformation and instabilities. Reduction of the EDFA pump power leads to a degradation in pulse SMSR, but this relationship is heavily dependent on the operating wavelength, and its position relative to the gain curve of the EDFA and FP laser being self-seeded. For example, at an operating wavelength of $1560 \mathrm{~nm}$, where the

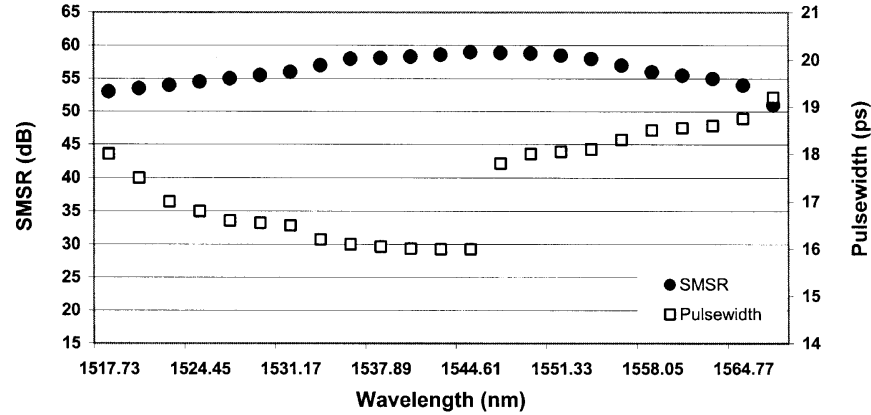

Fig. 4. SMSR (left-hand-side axis) and deconvolved pulsewidth (right-handside axis) against tunable range in the wavelength.

SMSR is $56 \mathrm{~dB}$ with $20-\mathrm{mW}$ EDFA pump power, the SMSR is reduced to $49 \mathrm{~dB}$ and $44 \mathrm{~dB}$ at pump powers of 15 and $10 \mathrm{~mW}$, respectively. Also presented in Fig. 4 is the pulsewidth variation as the wavelength is tuned. The point where the pulsewidth exhibits a sudden increase is the juncture when the seeded wavelength is moved from FP 1 to FP 2.

\section{CONCLUSION}

The generation of widely tunable $(\sim 50 \mathrm{~nm})$ self-seeded gainswitched short optical pulses that exhibit very high SMSR (in the order of $50 \mathrm{~dB}$ ) has been demonstrated. Such pulses (widely tunable and high SMSR) play a vital part in ensuring the optimal performance of high-speed WDM/OTDM optical communication networks [7]. Using an integrated dual laser source, it may be possible to develop a compact and highly stable SSGS wavelength tunable pulse source suitable for use in future high-speed optical networks.

\section{REFERENCES}

[1] C.-K. Chan, K. L. Sherman, and M. Zirngibl, "A fast 100-channel wavelength-tunable transmitter for optical packet switching," IEEE Photon. Technol. Lett., vol. 13, pp. 729-731, July 2001.

[2] R. Ludwig, U. Feiste, E. Dietrich, H. G. Weber, D. Breuer, M. Martin, and F. Küppers, "Experimental comparison of $40 \mathrm{Gbit} / \mathrm{s} \mathrm{RZ}$ and NRZ transmission over standard single mode fiber," Electron. Lett., vol. 35, pp. 2216-2218, 1999.

[3] T. Morioka, H. Takara, S. Kawanishi, O. Kamatani, K. Takiguchi, K. Uchiyama, M. Saruwatari, H. Takahashi, M. Yamada, T. Kanamori, and H. Ono, "1 Tbit/s (100 Gbit/s times 10 channel) OTDM/WDM transmission using a single supercontinuum WDM source," Electron. Lett., vol. 32, pp. 906-907, 1996

[4] L. P. Barry, R. F. O'Dowd, J. Debeau, and R. Boittin, "Tunable transform limited pulse generation using self-injection locking of an FP laser," IEEE Photon. Technol. Lett., vol. 5, pp. 1132-1134, Oct. 1993.

[5] D. Huhse, M. Schell, W. Utz, J. Kassner, and D. Bimberg, "Dynamics of single-mode formation in self-seeded Fabry-Perot laser diodes," IEEE Photon. Technol. Lett., vol. 7, pp. 351-353, Apr. 1995.

[6] L. P. Barry and P. Anandarajah, "Effect of side mode suppression ratio on the performance of self-seeded, gain-switched optical pulses in lightwave communications systems," IEEE Photon. Technol. Lett., vol. 11, pp. 1360-1363, Nov. 1999.

[7] P. Anandarajah, L. P. Barry, and A. Kaszubowska, "Performance issues associated with WDM optical systems using self-seeded gain-switched pulse sources due to mode partition noise," IEEE Photon. Technol. Lett., vol. 14, pp. 1202-1204, Aug. 2002.

[8] J. W. Chen and D. N. Wang, "Self-seeded gain switched optical short pulse generation with high side mode suppression ratio and extended wavelength tuning range," Electron. Lett., vol. 39, pp. 679-681, 2003.

[9] X. Fang and D. N. Wang, "Mutual pulse injection seeding by the use of two Fabry-Perot laser diodes to produce wavelength tunable optical short pulses," IEEE Photon. Technol. Lett., vol. 15, pp. 855-857, June 2003. 